\title{
Application of quality nursing to patients with cerebral thrombosis.
}

\author{
Zong-Yin Peng, Guo-Li Long* \\ Department of the Intensive Care Unit, East Branch, Sichuan Academy of Medical Sciences and Sichuan Provincial \\ People's Hospital, Chengdu, Sichuan, PR China
}

\begin{abstract}
Objectives: This study aims to explore the application of quality nursing to patients with cerebral thrombosis and determine its clinical effects on the disease.

Methods: A total of 131 patients with cerebral thrombosis admitted between April 2015 and July 2017 to the hospital where the author works were adopted as experimental subjects. Odd-even method was used to divide the patients into the control (66 cases) and test groups ( 65 cases), which were given with route and quality nursing, respectively. Finally, the two groups were compared in terms of overall response rate, satisfaction degree, Modified Barthel Index (MBI) rating, and hospitalization length.

Results: The overall response rate in the control group was $81.82 \%$, which significantly increased to $\mathbf{9 8 . 4 6 \%}$ in the test group $(\mathrm{P}<\mathbf{0 . 0 5})$. The patients' satisfaction degree in the control group administered with route nursing was $77.27 \%$, which significantly improved to $98.46 \%$ in the test group $(P<0.05)$. The MBI rating also significantly improved in the test group $(\mathbf{P}<0.05)$. Moreover, the length of hospitalization was significantly $(\mathbf{P}<\mathbf{0 . 0 5})$ shortened in the test group compared with that in the control group.

Conclusions: Quality nursing is vital to clinical nursing of patients with cerebral thrombosis. This study verified the clinical effects of quality nursing on cerebral thrombosis. Results showed that quality nursing could improve the patients' daily living activity and nursing satisfaction degree and shorten their length of hospitalization. These clinical effects of quality nursing can help optimize the prognosis of patients with cerebral thrombosis and contribute to their recovery.
\end{abstract}

Keywords: Cerebral thrombosis, Quality nursing, Clinical effects.

Accepted on October 24, 2017

\section{Introduction}

During atherosclerosis of cerebral artery, which is responsible for brain blood supply, the lumens narrow down, the blood vessels thicken, and blood clots are formed in the artery [1]. All these symptoms manifest cerebral thrombosis, which can considerably harm the human body, especially in terms of limb and language functions [2]. As such, this condition leads to high disability and fatality rates. This study aims to clarify the optimal nursing model for patients with cerebral thrombosis and determine the value of quality nursing model.

\section{Materials and Methods}

\section{Data}

A total of 131 patients with cerebral thrombosis admitted to our hospital between April 2015 and July 2017 were included in the study. Odd-even method was used to divide the patients into control and test groups. The control group comprised 66 cases including 36 males and 30 females, aging from 55 to 79 $\mathrm{y}$, with an average age of $65.1 \pm 9.33 \mathrm{y}$. The test group comprised 65 cases, with 39 males and 26 females, aging from
53 to $81 \mathrm{y}$, with an average age of $65.23 \pm 9.39 \mathrm{y}$. Patients in the two groups were compared in terms of sex and age structure. Results showed no significant difference $(\mathrm{P}>0.05)$.

\section{Methods}

Psychological nursing: Cerebral thrombosis is often accompanied by conscious disturbance, dizziness, memory impairment, limb numbness, and aphasia. Patients with cerebral thrombosis also suffer from a poor psychological state. All of these conditions can reduce treatment compliance. To resolve this issue, nursing personnel should initiate communication with patients suffering from cerebral thrombosis to learn their nursing demands. In this manner, the nursing personnel can provide appropriate nursing services to the patients, particularly effectively helping them improve their psychological state.

Life nursing: Limb numbness is a complication of cerebral thrombosis. Patients suffering from limb numbness may have impeded daily living activity. Therefore, nursing personnel should provide life nursing services to patients with cerebral thrombosis according to their practical situations [3,4]: (1) the nursing personnel should ensure that the patients lie on the bed 
in a comfortable posture and help them turn over or pat them on their back at fixed time; (2) the patients might fall down from the bed because of inconvenience in moving around and disturbance due to consciousness; hence, the nursing personnel should meticulously implement relevant precautions; and (3) the rationality and purposefulness of dieting should be guaranteed based on the physical conditions of the patients. The nursing personnel should properly increase the intake of vegetables, fruits, meat, eggs, and milk and guarantee nutritional balance.

Nursing of complications: When patients suffer from cerebral thrombosis, various complications, including pulmonary infection, respiratory tract infection, and spasm, can occur. The nursing personnel should pay attention to the respiratory tract, oral cavity, anti-infection, and expectoration when providing nursing services. The respirator should be properly prepared for therapeutic intervention if a patient suffers from a respiratory disturbance. The nursing personnel should demonstrate to the patient the correct way of coughing. The nursing personnel should adjust the patient to achieve comfortable posture and avoid symptoms of spasms if a patient has limb disturbance. The nursing personnel should reasonably perform anti-infection nursing for a patient inserted with a catheter for indwelling.

Performance of rehabilitation training: During the progression of cerebral thrombosis, limb and language dysfunctions commonly occur among the patients. In this regard, the symptoms of patients with cerebral thrombosis should be observed. Limb and language function rehabilitation trainings should be planned accordingly based on the symptoms of the patients. The designed plans should guarantee their targeted effects. Function rehabilitation training, either passive or active, should be properly conducted on the bed for a patient manifesting difficulty in getting out of the bed for relevant activities. If a patient can move around independently, then the nursing personnel should increase the patient's frequency of activities on the ground and periodically pat on the dysfunctional joints and muscles of the patient. The nursing personnel should also provide guidance for the patient's sound production and reasonably implement language rehabilitation training if a patient encounters language dysfunction.

\section{Criteria}

Criteria of curative effects: When nursing is administered to the patients, "markedly effective" means that the patients' life indices are all normal and the disease is stable; "effective" means that the patients' life indices are normal and the disease is relatively stable; and "failed" means that the patients' life indices are abnormal and the disease is unstable.

Criteria of daily living activity: The daily living activity of patients with cerebral thrombosis in the two groups was rated using the Modified Barthel Index (MBI) scale. The full marks were 100 . The final rating results were positively correlated with the daily living activity of the patients.
Criteria of nursing satisfaction degree: A questionnaire for assessing nursing satisfaction degree was distributed among patients with cerebral thrombosis admitted to the hospital where the author works. The final questionnaire survey results were positively correlated with the nursing satisfaction degree of patients with cerebral thrombosis. The satisfaction degree was scored based on a 100 marking system [5]: strongly satisfying: $\geq 90$ points; generally satisfying: $\geq 70$ but $<90$; and strongly dissatisfying: $<70$.

\section{Statistical methods}

SPSS20.0 was used for statistical analysis of the clinical effects of routine and quality nursing administered to the two groups of patients with cerebral thrombosis. T-test was performed for group comparison of measurement data (including MBI rating results) presented in the form of $\bar{x} \pm S$. $\chi^{2}$-test was carried out for group comparison of enumeration data (overall response rate) presented in the form of $(\%)$. The difference was considered statistically significant at the level of $0.05(\mathrm{P}<0.05)$.

\section{Results}

\section{Comparison of the overall response rate}

Table 1 shows that the overall response rate in the test group (98.46\%) was significantly improved compared with that in the control group $(81.82 \%)(\mathrm{P}<0.05)$.

Table 1. Clinical comparison of the overall response rate of patients with cerebral thrombosis in the control and test groups.

\begin{tabular}{llllll}
\hline Group & $\mathbf{n}$ & $\begin{array}{l}\text { Markedly } \\
\text { effective }\end{array}$ & Effective & Failed & $\begin{array}{l}\text { Overall response } \\
\text { rate (\%) }\end{array}$ \\
\hline Test group & 65 & 57 & 7 & 1 & 98.46 \\
\hline $\begin{array}{l}\text { Control } \\
\text { group }\end{array}$ & 66 & 45 & 9 & 12 & 81.82 \\
\hline $\mathrm{X}^{2}$ & & & & 10.1481 \\
\hline $\mathrm{P}$ & & & & 0.0014 \\
\hline
\end{tabular}

\section{Comparison of nursing satisfaction degree}

As shown in Table 2, the nursing satisfaction degree in the control group was $77.27 \%$, which is lower than that in the test group $(98.46 \%)(\mathrm{P}<0.05)$.

Table 2. Clinical comparison of nursing satisfaction degree of patients with cerebral thrombosis in the control and test groups.

\begin{tabular}{llllll}
\hline Group & $\mathbf{n}$ & $\begin{array}{l}\text { Strongly } \\
\text { satisfying }\end{array}$ & $\begin{array}{l}\text { Generally } \\
\text { satisfying }\end{array}$ & $\begin{array}{l}\text { Dissatisfyin } \\
\mathbf{g}\end{array}$ & $\begin{array}{l}\text { Overall } \\
\text { satisfaction } \\
\text { percentage (\%) }\end{array}$ \\
\hline Test & 65 & 57 & 7 & 1 & 98.46 \\
\hline Control & 66 & 41 & 10 & 15 & 77.27 \\
\hline $\mathrm{X}^{2}$ & & & & & 13.7127 \\
\hline
\end{tabular}




\begin{tabular}{ll}
\hline $\mathrm{P}$ & 0.0002 \\
\hline
\end{tabular}

\section{Comparison of the $\mathrm{MBI}$ rating}

Table 3 demonstrates that the MBI rating in the test group was significantly $(\mathrm{P}<0.05)$ improved compared with that in the control group.

Table 3. Clinical comparison of the MBI rating of the patients with cerebral thrombosis in the control and test groups $(\bar{x} \pm S$, points).

\begin{tabular}{llll}
\hline Group & $\mathbf{n}$ & Before nursing & After nursing \\
\hline Test group & 65 & $32.92 \pm 4.29$ & $75.82 \pm 3.33$ \\
\hline Control group & 66 & $32.47 \pm 4.36$ & $62.95 \pm 3.15$ \\
\hline $\mathrm{t}$ & & 0.5953 & 22.7275 \\
\hline $\mathrm{P}$ & 0.5526 & 0.0000 \\
\hline
\end{tabular}

\section{Comparison of the length of hospitalization}

As shown in Table 4, the length of hospitalization of patients with cerebral thrombosis in the test group was significantly $(\mathrm{P}<0.05)$ shortened compared with that in the control group.

Table 4. Clinical comparison of the length of hospitalization of patients with cerebral thrombosis in the control and test groups $(\bar{x} \pm$ $S$, days).

\begin{tabular}{lll}
\hline Group & $\mathbf{n}$ & Length of hospitalization \\
\hline Test group & 65 & $15.66 \pm 4.05$ \\
\hline Control group & 66 & $24.85 \pm 4.85$ \\
\hline $\mathrm{t}$ & & 11.7625 \\
\hline $\mathrm{P}$ & $<0.05$ \\
\hline
\end{tabular}

\section{Discussion}

Cerebral thrombosis, which falls under the category of ischemic cerebrovascular diseases, often occurs among elders. The pathogenesis of cerebral thrombosis is frequent. Negative factors, including coronary heart diseases, hypertension, diabetes, and unhealthy dietary habit, can lead to cerebral thrombosis [6,7]. Traditional Chinese medical treatment and physiotherapy are commonly adopted for patients with cerebral thrombosis. Although these methods can improve the symptoms of the disease, they can easily trigger a series of complications among patients who already suffered severely from cerebral thrombosis [8]. Therefore, an effective nursing model must be developed.

The comprehensiveness and efficiency of quality nursing for patients with cerebral thrombosis should be guaranteed. The nursing personnel should determine the nursing needs of patients and provide immediate and targeted nursing services to them. Complete implementation of quality nursing can significantly contribute to the rehabilitation of patients with cerebral thrombosis, leading to significantly improved overall nursing effects [9]. In addition, quality nursing can gradually alleviate the patients' sense of fear and anxiety, resulting in increased treatment compliance of the patients and strengthened confidence in treatment effects. Benefiting from all these clinical effects of quality nursing, the cerebral thrombosis nursing satisfaction degree and the overall nursing effects of the patients can be significantly improved [10].

In this research, the overall response rate in the control group was $81.82 \%$, which significantly increased to $98.46 \%$ in the test group $(\mathrm{P}<0.05)$. The nursing satisfaction degree of patients in the control group was $77.27 \%$, which significantly increased to $98.46 \%$ in the test group $(\mathrm{P}<0.05)$. The $\mathrm{MBI}$ rating was also significantly improved in the test group $(\mathrm{P}<0.05)$. Moreover, the length of hospitalization in the test group was significantly $(\mathrm{P}<0.05)$ shortened compared with that in the control group. All these results provide substantial basis for the clinical value of quality nursing for patients with cerebral thrombosis.

\section{Conclusion}

The clinical application of quality nursing to patients with cerebral thrombosis can improve the daily living activity and nursing satisfaction degree and shorten the length of hospitalization among the patients. These clinical effects can guarantee the improvement of cerebral thrombosis nursing effects and facilitate cerebral thrombosis treatment.

\section{References}

1. Wang B, Yee Aw T, Stokes KY. N-acetylcysteine attenuates systemic platelet activation and cerebral vessel thrombosis in diabetes. Redox Biol 2017; 14: 218-228.

2. Li ZR. Cerebral hemorrhage treatment drug edaravone showed inhibition effect towards Drug-Metabolizing Enzymes (Dmes). Lat Am J Pharm 2017; 36: 1328-1330.

3. Taniguchi D, Nakajima S, Hayashida A, Kuroki T, Eguchi H, Machida Y, Hattori N, Miwa H. Deep cerebral venous thrombosis mimicking influenza-associated acute necrotizing encephalopathy: a case report. J Med Case Rep 2017; 11: 281.

4. Wu FY, Jiang BW, Li B, Meng L, Zhu GF, Wu JX. Endocrine disrupting chemical Bisphenol AF (BPAF) affects the metabolic elimination of cerebral hemorrhage inducing drugs. Lat Am J Pharm 2017; 36: 1304-1307.

5. Kulkarni GB, Singh RJ, Gadad V, Ramakrishnan S, Mustare V. Unilateral Papilledema in Cerebral Venous Sinus Thrombosis. J Neurosci Rural Pract 2017; 8: S106S110.

6. Goyal K, Agarwal R. Pulse based sensor design for wrist pulse signal analysis and health diagnosis. Biomed Res India 2017; 28: 5187-5195.

7. Kawahara I, Toyoda K, Hirose M, Kitagawa N. A case of cerebral venous sinus thrombosis, secondary to subclinical basedow disease. No Shinkei Geka 2017; 45: 805-810.

8. Kartal A. Facial palsy in cerebral venous thrombosis: an atypical case in a young girl. J Pediatr Neurosci. 2017; 12: 160-161. 
9. Preethi DMD, Jayanthi VE. SOM clustering approach: investigation on cataract surgery structural changes in iris. Biomed Res India 2017; 28: 5210-5216.

10. Czekirda M, Chrusciel P, Czekirda N, Jarosz MJ. Psychosocial aspect of quality of life among working and unemployed nurses and midwives. Ann Agric Environ Med 2017; 24: 472-476.

\section{*Correspondence to}

Guo-Li Long

Department of the Intensive Care Unit

East Branch

Sichuan Academy of Medical Sciences and Sichuan Provincial People's Hospital

PR China 